\title{
La gestión de los recursos humanos en las fuerzas de ventas, un estudio exploratorio a través del Método Delphi aplicado a las empresas \\ peruanas
}

\section{Human resources management in the sales forces, an exploratory study through the Delphi method applied to Peruvian companies}

${ }^{1}$ Candidato a Doctor en Administración de Empresas por la Universidad de Lleida, España. Master en Business Administration, Universidad Adolfo Ibañez, Chile. Investigador, Facultad de Economía y Negocios, Universidad de Desarrollo, Chile. E-mail: j.bullemore@udd.cl

${ }^{2}$ Doctor en Economía por la Universitat de Lleida (España). Profesor del departamento de Administración de Empresas en la Universitat de Lleida (España) C/ Jaume II, 73, Lleida, España. E-mail: ecristobal@aegern.udl.cat 


\section{RESUMEN}

En este artículo se explora la forma como se desarrolla la gestión de las fuerzas de ventas en el Perú desde la perspectiva de los recursos humanos. Su objetivo principal es el diagnóstico de aquellas variables de productividad comercial relacionadas con la gestión de personas tales como el reclutamiento y selección de vendedores, los roles que desempeñan los supervisores comerciales, y sobre cómo las firmas gestionan el desarrollo de las competencias específicas para ventas, para así proponer acciones estratégicas para gestores, consultores y académicos en general. Se desarrolló un estudio a través del método Delphi en dos rondas con un panel de expertos. Al final de este estudio se presentan una serie de implicaciones gerenciales centradas en el desarrollo de habilidades de liderazgo comercial.

PALABRAS CLAVE: Dirección comercial, Método Delphi, Ventas, Investigación Cualitativa, Perú.

\section{ABSTRACT}

This article explores the way in which sales forces are managed in Peru from the perspective of human resources $(\mathrm{HR})$. Its main objective is the diagnosis of those variables of sales productivity related to HR such as the recruitment and selection of salespersons, the roles played by first line supervisors, and how firms manage the development of specific competencies for sales, to propose strategic actions for managers, consultants and academics in general. A study was developed through the Delphi method in two rounds with a panel of experts. At the end of this study, a series of managerial implications are presented, focused on the development of commercial leadership skills.

KEYWORDS: Sales Management, Delphi Method, Sales, Qualitative Research, Perú. 


\section{INTRODUCCIÓN}

En la academia latinoamericana existe una escasa investigación sobre temáticas comerciales y de ventas (Campbell \& Fransi 2016), no obstante, lo anterior su investigación alcanza enormes oportunidades para las empresas (Baldauf \& Lee, 2011), y en particular para países de mercados emergentes latinoamericanos.

La investigación académica relacionada a la gestión de fuerzas de ventas en el Perú ha sido escasa. En Google Scholar tan solo se encuentran un puñado de artículos, entre los que destaca la investigación desarrollada en 2009 por Barat y Spillan respecto a la percepción de los estudiantes universitarios peruanos y guatemaltecos en desarrollar una carrera en ventas. A su vez, algunos estudios sobre prácticas en dirección comercial y ventas a nivel multicultural como son los estudios de Karakaya, Quigley y Bingham (2011), Hill, Still, y Boya (1991), Hill y Still (1990), Hill y Birdseye (1989), Hill y Allaway (1993), White, Absher, y Huggins (2011), Dawson (1994). Por último, se pueden encontrar algunas tesis de grado tales como el trabajo de Rojas y Tito (2015), Gutiérrez (2015) y Flores (2012).

El principal objetivo de esta investigación es poder explorar cómo las empresas peruanas realizan la gestión de los recursos humanos en sus fuerzas de ventas. Para tal efecto, se usará el método Delphi, pues permite obtener información y hacer uso de ella de un modo más rápido y eficiente que con los métodos tradicionales (Vélez, 2002). Para el desarrollo de este objetivo se reconocerán y valorizarán aquellas palancas de productividad comercial relacionadas con la gestión de los recursos humanos que actualmente se usan en las compañías peruanas. De esta forma se espera dar relevancia a aquellas variables que hoy no están siendo bien atendidas por la academia y por los directivos de empresas en el Perú.

En el desarrollo de la presente investigación se busca como objetivo operativo el entender cómo las empresas del Perú gestionan sus recursos humanos comerciales en relación a:

- Los roles de los supervisores comerciales. 
- El reclutamiento, selección y rotación de los vendedores.

- Formación y capacitación comercial.

Para dar cumplimiento a estos objetivos, se examinará la literatura sobre la dirección comercial y de ventas, para así plantear las diversas hipótesis. A continuación, se expone la metodología utilizada y se comentan los resultados del estudio mostrarando los principales desafíos, tendencias y problemas en el sector de las ventas en el Perú. Finalmente, se presentan las principales conclusiones y sus implicaciones en la gestión de empresas.

\section{REVISIÓN BIBLIOGRÁFICA}

Siguiendo el trabajo desarrollado por Bullemore y Cristóbal (2016) se estudiaron los siguientes factores de la gestión de los recursos humanos: reclutamiento y selección del personal de ventas; capacitación, entrenamiento y desarrollo de habilidades; y gestión de la rotación de empleados.
Hay una escasez de vendedores calificados a nivel global (Nielson, Eichmeier y Cooper-Larsen 2017). La formación se produce en etapas tempranas en las carreras de los directivos de ventas (Powers, DeCarlo \& Gupte, 2010). El interés de candidatos en buscar trabajos de ventas se ve afectado por la terminología utilizada en las descripciones de cargos, donde la terminología enfocada en las necesidades del cliente aumenta el interés en buscar un trabajo de ventas (Kim y Talbott, 2018). De esta manera, que la necesidad de técnicas inteligentes contratación y retención de empleados en un mercado global altamente competitivo ha crecido significativamente en la última década (Khosla, Goonesekera, y Chu, 2009). Existe una gran importancia en las políticas de reclutamiento y selección del personal de ventas, así como la contratación de un nuevo vendedor podría afectar la calidad y el desempeño de una fuerza de ventas completa, y también la rotación de vendedores (Darmon, 2008). Existen varios factores que explicarían la variabilidad en un exitoso desempeño en ventas tal como la selección de vendedores (Cron, Marshall, Singh, Spiro, \& Sujan, 2005). Para lograr aumentos en 
el desempeño comercial la organización deberá comenzar a reemplazar al personal de ventas con procedimientos de selección válidos (Farrell \& Hakstian, 2001).

En las últimas décadas los investigadores en dirección estratégica de recursos humanos han estudiado las razones que impulsan el cumplimiento de sus objetivos financieros, comerciales y estratégicos a través del uso capacitación, formación y desarrollo de competencias de sus colaboradores, también disminución de la rotación de empleados, y aumentos de productividad en diversos ámbitos como la calidad, servicio, y el desempeño financiero (Jiang et al., 2012). A su vez, se han producido cambios significativos que han afectado a la función de ventas y las competencias necesarias de los gerentes de ventas. Si los vendedores creen que tienen la capacidad para realizar las tareas necesarias para el éxito de ventas, entonces ese vendedor estará satisfecho con el entrenamiento. Además, si el vendedor tiene confianza en su capacidad de utilizar el conocimiento del producto, entonces él o ella tendrá más probabilidades de estar satisfecho con la formación del producto. Por lo tanto, los esfuerzos iniciales de formación deben concentrarse en el seguimiento de la autoeficacia. El entrenamiento de ventas necesita ser dirigido a la mejora de competencias y creencias. Las organizaciones de ventas pueden ayudar a los nuevos vendedores proporcionando mentores durante el entrenamiento $y$ tratando de desarrollar y fomentar un clima de entrenamiento favorable (Sager, Dubinsky, Wilson y Shao, 2014). Existe una brecha importante en la comprensión de los gerentes de ventas con respecto a la evaluación de la formación de habilidades de ventas. Este problema se ve agravado por la ausencia de un marco integrado que sintetice y explique los múltiples niveles de evaluación (Attia, Honeycutt \& Fakhr, 2012). Las organizaciones reportan grandes dificultades para medir con precisión el talento, lo que refleja la falta de fundamentos teóricos para el talento de su identidad en la literatura la gestión estratégica de los recursos humanos (Nijs, Gallardo, Dries \& Sels, 2013). En síntesis, el entrenamiento de competencias y desarrollo de habilidades en ventas es muy relevante para mejoras estratégicas en la gestión de los recursos humanos. 
Desde hace más de un siglo, compañías de todo el mundo han usado comisiones y bonos para motivar y dirigir la gestión, y la actividad de las fuerzas de ventas. No obstante, lo anterior y dado que la venta se vuelve cada vez más compleja, la motivación de la fuerza de ventas se vuelve más difícil de determinar (Zoltners et al. 2012). Sin lugar a dudas la motivación es un componente fundamental para cualquier modelo de gestión del desempeño, y se puede separar en intrínseca o extrínseca. De esta manera los motivadores extrínsecos corresponden a instrumentos de ganancia o pérdida, como por ejemplo un sistema de incentivos. Los motivadores intrínsecos tienen relación con el compromiso del empleado (Cerasoli, Nicklin \& Ford, 2014). Investigadores de la gestión de recursos humanos han defendido con fuerza un sistema de gestión del desempeño dando perspectiva y proporcionado pruebas de que ciertos sistemas tienen un impacto significativo en el desempeño individual y organizacional. Sin embargo, se han hecho pocos esfuerzos para explicar el ajuste interno entre los sistemas de recursos humanos y cómo las prácticas trabajan en conjunto (Jiang, Lepak, Han, Hong, Kim y Winkler, 2012). La colaboración de ventas se relaciona positivamente con los resultados empresariales, mientras que el individualismo está negativamente relacionado con la colaboración de las ventas. Un plan de recompensas por alineamiento conduce a una mayor colaboración de ventas y es particularmente importante en las sociedades altamente individualistas (Magnusson, Peterson y Westjohn, 2014). Existe una correlación positiva entre la satisfacción de los empleados, la satisfacción del cliente y el valor para los accionistas. La satisfacción y la lealtad del cliente continuará mientras los empleados estén satisfechos y que la compañía entregue la calidad esperada por el cliente. De esta manera la satisfacción del cliente y la satisfacción de los empleados aumentarán el valor para los accionistas (Saeed, Zehou, Hussain y Anwar ul Haq, 2014). Los mejores vendedores se sienten atraídos por las organizaciones que proporcionan oportunidades para hacer pleno uso de sus capacidades. Esta variable es fundamental para las ventas ya que incremente el rendimiento, y disminuye la rotación y estrés de los vendedores (Jaramillo, Mulki y Boles, 2013). La investigación sobre la relación 
entre el clima ético y la satisfacción en el trabajo es emergente en la literatura (Wang y Hsieh, 2012). El desarrollo de un clima de trabajo ético es importante para las organizaciones. Así la justicia organizacional, distributiva y la justicia procesal, tienen una relación directa en la percepción de los vendedores respecto del clima de trabajo ético y de su compromiso con la organización (Deconinck, Johnson \& Busbin, 2012).

Dados los conceptos revisados se considera necesaria una investigación que revele un primer diagnóstico, y aporte información sobre los principales desafíos, tendencias y problemas en el sector de las ventas.

\section{MÉTODO}

Se desarrolló el análisis usando el modelo desarrollado por Zoltners, Sinha y Lorimer (2008), el cual ha sido ampliamente usado en la academia, tal como se puede apreciar en artículos como los publicados por Albers, Mantrala, y Sridhar (2010), Misra y Nair (2011), Chung, Steenburgh, y Sudhir (2013), Mantrala, et al. (2010), Bolander, Dugan, y Jones (2017) entre muchos otros. Según plantean sus autores el modelo sirve para organizar las complejidades de la función de ventas, y ayuda a diagnosticar oportunidades de mejora e investigación (ver figura 1). 


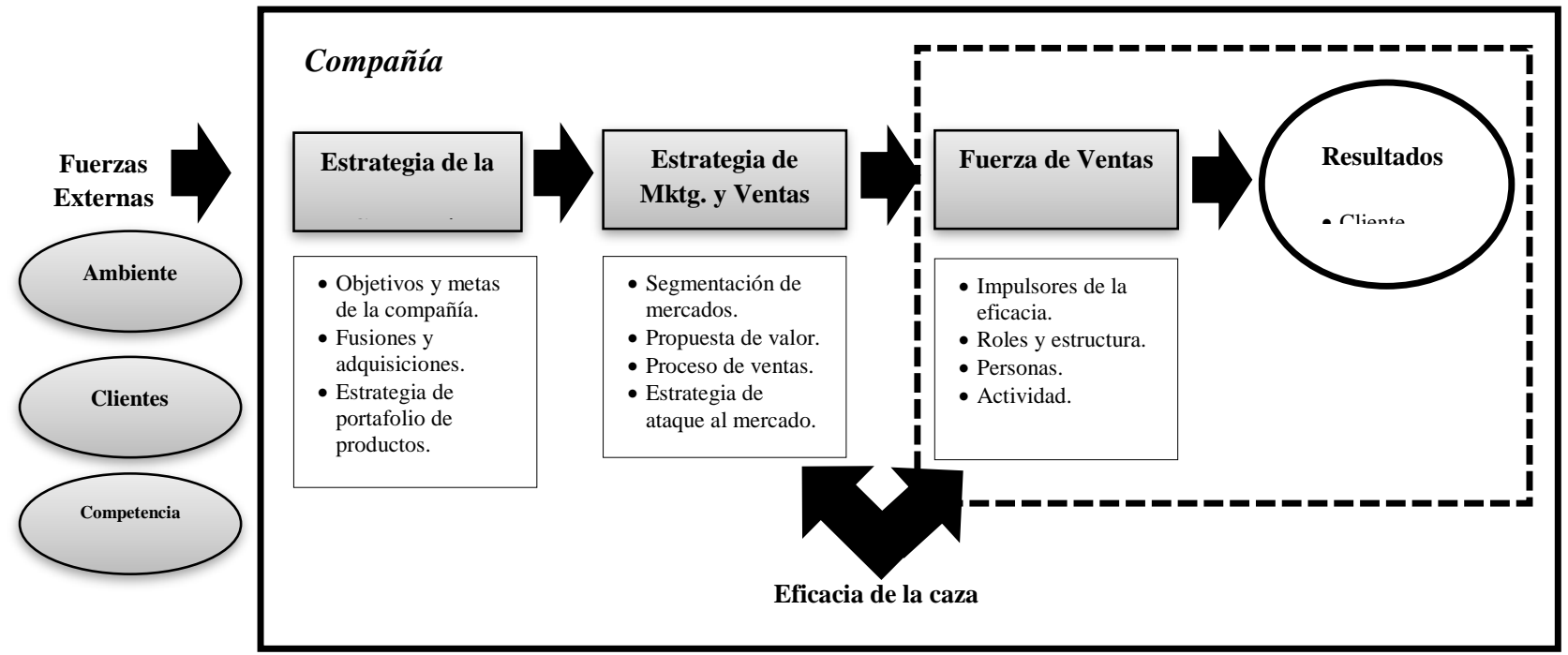

Figura 1. Modelo de efectividad de la fuerza de ventas Fuente: Zoltners et al. (2008, p. 117)

Dentro del modelo se señala la importancia de las personas que componen la fuerza de ventas, por lo que se plantean las siguientes hipótesis:

H1: Los supervisores de ventas no gestionan bien sus roles relacionados a la gestión de los recursos humanos.

H2: El reclutamiento, selección y rotación de los vendedores no se desarrolla de manera sistemática ni planificada.

H3: Las grandes empresas realizan una formación más planificada y sistemática que en las pequeñas y medianas empresas.
Además del modelo recién descrito, esta investigación usará los pasos formulado en el artículo "Estudio sobre el estado del reclutamiento y selección fuerzas de ventas a través de un panel de expertos en Chile" (Campbell y Fransi 2016), el cual desarrolla una investigación exploratoria a través del método Delphi. Esto se justifica en el carácter exploratorio de la investigación, pues no existe una información clara y evidente de base. De manera que utilizando este método se puede obtener dicha información y hacer uso de ella de un modo más rápido y eficiente que con los métodos tradicionales (Vélez, 2002). Originalmente fue desarrollado por Rand Corporation 
para el pronóstico tecnológico, esta técnica recibió su nombre del famoso oráculo de Delphi (Hasson, Keeney y McKenna 2000).

Según Loo (2002), el método Delphi presenta cinco características fundamentales 1) La muestra está compuesta por un grupo de expertos cuidadosamente seleccionados que representan un amplio espectro de opinión del objeto de estudio. En esta investigación se ha seleccionado a expertos con diversos perfiles. 2) El anonimato es fundamental pues ningún experto debe conocer la identidad de los otros que componen el grupo de debate. Esta característica impide la posibilidad de ser influenciado por la mayoría o por miembros relevantes del grupo. También permite que un miembro cambie sus opiniones sin que eso suponga una pérdida de imagen. $Y$, además, el experto puede defender sus argumentos con la tranquilidad de que sus equivocaciones no son conocidas por el resto. Lo anterior se respetó en todo momento. 3) Existe un moderador o investigador que construye y distribuye los cuestionarios e informes de resultados durante todo el proceso del Delphi, en el caso de esta investigación los moderadores fueron los autores del artículo. 4) La iteración es fruto de las diferentes circulaciones que se realizan con los cuestionarios. Al presentar los resultados obtenidos con los cuestionarios, se consigue que los expertos conozcan los distintos puntos de vista, de esta manera, cada experto puede reconsiderar su opinión si los argumentos presentados le parecen más apropiados que los suyos. Vale la pena mencionar que este paso también fue desarrollado por el equipo investigador. 5) El resultado final es un informe de investigación donde aparecen las respuestas, planes y estrategias que han sugerido los miembros del panel.

En la literatura de la gestión de empresas hay relativamente pocos trabajos que se hayan hecho uso de este método. Se pueden nombrar algunos como los desarrollados por Basu \& Schroeder (1977), Kauko \& Palmroos (2014), Mitra (2013), Småros \& Hellström (2004), Gupta \& Clarke (1996), Wood \& Mauskopf (1991), Chang \& Wang (2006), Rowe \& Wright (1999), Campos et al. (2014), Bonnemaizon, Cova y Louyot (2007), Hayes (2007), Cabero Almenara e Infante Moro (2014) 


\section{DESARROLLO/ANÁLISIS/RESULTADO}

Las rondas Delphi fueron realizadas entre los meses de marzo y abril de 2018. Durante el mes de marzo de 2018 se eligió un total de veinte personas para conformar el panel de expertos. El promedio de edad del panel de expertos elegido era de 40,6 años, con un rango de 30 años a 51 años. A la fecha del estudio más del $90 \%$ contaba con más de diez años de experiencia profesional, y al menos el grado académico de licenciado, destacando que sobre el $60 \%$ tenía el grado de master. A continuación, se realizó la primera fase del cuestionario online entre los días 15 de marzo y el 4 de abril de 2018, del que se obtuvieron un total diez y seis respuestas completas, una incompleta, y tres personas no contestaron. La segunda etapa fue realizada entre los días 5 de abril y el 13 de abril de 2018, obteniendo un total de diez y seis respuestas completas, una incompleta, cuatro personas que no contestaron (ver tabla 1).

Tabla 1: Demografía del panel de expertos

\begin{tabular}{|l|c|c|}
\hline Total respuestas & Primera ronda Delphi & Segunda ronda Delphi \\
\hline Respuestas completas & $16(100,0 \%)$ & $16(100,0 \%)$ \\
\hline Perfil & Primera ronda Delphi & Segunda ronda Delphi \\
\hline Directivos de empresas & $16(100,0 \%)$ & $16(100,0 \%)$ \\
\hline Experiencia & Primera ronda Delphi & Segunda ronda Delphi \\
\hline Entre 5 y 10 años & $4(25,0 \%)$ & $6(37,5 \%)$ \\
\hline 10 años o más & $12(75,0 \%)$ & $10(62,5 \%)$ \\
\hline Total & $16(100 \%)$ & $16(100 \%)$ \\
\hline Mundo Corporativo & Primera ronda Delphi & Segunda ronda Delphi \\
\hline Consultoría & $5(31,25 \%)$ & $4(25,0 \%)$ \\
\hline Sector Financiero & $3(18,75 \%)$ & $5(31,25 \%)$ \\
\hline Industrial & $4(25,0 \%)$ & $4(25,0 \%)$ \\
\hline Consumo masivo & $4(25,0 \%)$ & $3(18,75,4 \%)$ \\
\hline Total & $16(100 \%)$ & $16(100 \%)$ \\
\hline Grado académico & Primera ronda Delphi & Segunda ronda Delphi \\
\hline Licenciado & $3(18,75 \%)$ & $2(12,5 \%)$ \\
\hline Master & $13(81,25 \%)$ & $14(87,5 \%)$ \\
\hline Total & $16(100 \%)$ & $16(100 \%)$ \\
\hline
\end{tabular}

Fuentes: propias 
Por su grado de aceptación y uso en cuestionarios para la investigación, principalmente en ciencias sociales se escogió el uso de la escala de Likert. Se solicitó al experto su opinión en relación a distintos factores de productividad de las fuerzas de ventas en el Perú. Para ello se le solicitó marcar únicamente en la columna que considera que se ajusta mejor a la realidad, siendo el 1) No estoy de acuerdo; 2) Poco de acuerdo; 3) Ni de acuerdo, ni en desacuerdo; 4) Bastante de acuerdo; 5) Muy de acuerdo. Las hipótesis se darán como validadas en la medida que la mediana se aleje en al menos medio punto de la respuesta 3) Ni de acuerdo, ni en desacuerdo.

De la primera etapa de estudio se puede extraer que existen varios temas con un gran consenso entre los expertos, sin embargo, en otros no existe claridad, pues el valor final es cercano al valor tres (3: ni de acuerdo ni en desacuerdo). Por lo tanto, en la segunda etapa del Delphi se intentó lograr el acuerdo dentro del panel de expertos en dichos temas, como también abordar nuevos aspectos a petición de los comentarios recibidos durante la primera etapa.
Siguiendo los trabajos de Calabuig \& Crespo (2009); Gil-Gómez de Liaño \& Pascual-Ezama (2012); y Taleghani et al. (2011) entre otros se eligieron complementariamente dos medidas estadísticas para poder interpretar los resultados: el Promedio y la Mediana. Al ser medidas ampliamente conocidas y utilizadas por la academia no serán descritas.

La encuesta fue estructurada en varios apartados en función de la temática abordada. Los apartados eran los siguientes: a) definición de roles de los supervisores de ventas en el Perú; b) reclutamiento, selección y rotación del personal de ventas; c) desarrollo de competencia en ventas.

\section{Definición de roles de los}

\section{supervisores de ventas en el Perú}

En esta fase los expertos aportaron los siguientes comentarios: "Hay una necesidad de desarrollar mayor y mejor gestión de liderazgo enfocada en desarrollo humano y del talento. Muchos profesionales llegan a la función de venta por una necesidad de trabajo y no 
necesariamente por vocación." Los

resultados de este apartado se

encuentran reflejados en las tablas 2 y 3 .

Tabla 2: Resultados de la primera ronda Delphi: definición de roles de los supervisores de ventas en el Perú

\begin{tabular}{|l|c|c|}
\hline Preguntas & Promedio & Mediana \\
\hline $\begin{array}{l}\text { Los supervisores de ventas en el Perú destinan el suficiente tiempo para } \\
\text { realizar reclutamiento y selección de vendedores. }\end{array}$ & 2,81 & 3 \\
\hline $\begin{array}{l}\text { Los supervisores de ventas en el Perú guían a sus dirigidos en } \\
\text { oportunidades de ventas. }\end{array}$ & 3,38 & 3,5 \\
\hline $\begin{array}{l}\text { Los supervisores de ventas en el Perú desarrollan habilidades de ventas } \\
\text { en sus dirigidos. }\end{array}$ & 2,75 & 3 \\
\hline $\begin{array}{l}\text { Los supervisores de ventas en el Perú facilitan el desarrollo de una cultura } \\
\text { corporativa orientada en el cliente. }\end{array}$ & 2,94 & 3 \\
\hline \begin{tabular}{l} 
TOTAL \\
\hline
\end{tabular}
\end{tabular}

Fuentes: propias

Sobre esta primera parte del estudio solo se puede concluir que los supervisores de ventas en el Perú guían a sus dirigidos en oportunidades de ventas. No quedan evidencias suficientes para validar nuestra primera hipótesis. Complementariamente alguno de los expertos realizó aportes tales como "Las empresas transnacionales bien estructuradas con operación en Perú tienen procesos políticos de ventas claras y definidas. En el caso de las empresas peruanas pequeñas y medianas son pocas las que tienen modelos de ventas definidos y documentados", también "En Perú las grandes empresas lo hacen, las medianas, una gran parte de ellas, tienden a realizarlo, pero las pequeñas y la otra parte de medianas, probablemente se basan en su experiencia, pero sin tenerlo físicamente descrito. 
Tabla 3: Resultados de la segunda ronda Delphi: definición de roles de los supervisores de ventas en el Perú

\begin{tabular}{|l|c|c|}
\hline Preguntas & Promedio & Mediana \\
\hline $\begin{array}{l}\text { Los supervisores de ventas en el Perú destinan el suficiente tiempo para } \\
\text { realizar reclutamiento y selección de vendedores. }\end{array}$ & 2,75 & 2 \\
\hline $\begin{array}{l}\text { Los supervisores de ventas en el Perú guían a sus dirigidos en } \\
\text { oportunidades de ventas. }\end{array}$ & 3,42 & 4 \\
\hline $\begin{array}{l}\text { Los supervisores de ventas en el Perú desarrollan habilidades de ventas } \\
\text { en sus dirigidos. }\end{array}$ & 2,83 & 2 \\
\hline $\begin{array}{l}\text { Los supervisores de ventas en el Perú facilitan el desarrollo de una cultura } \\
\text { corporativa orientada en el cliente. }\end{array}$ & 2,67 & $\mathbf{3}$ \\
\hline \begin{tabular}{l} 
TOTAL \\
\hline
\end{tabular}
\end{tabular}

Fuentes: propias

Una vez realizada la segunda etapa del proceso, se puede afirmar que los supervisores de ventas no destinan suficiente tiempo a reclutar y seleccionar vendedores. A su vez, guían bien a sus dirigidos en oportunidades de ventas. No queda definido si los supervisores ayudan a desarrollar las competencias profesionales de sus vendedores. Por último, los supervisores no facilitan el buen desarrollo de la cultura corporativa. Estos resultados confirman la primera hipótesis: Los supervisores de ventas no gestionan bien los roles relacionados a la gestión de los recursos humanos. Los expertos nos aportaron con el siguiente comentario "La mayor parte de supervisores de ventas en empresas medianas y pequeñas están mucho más orientados en lograr la meta mensual para obtener sus comisiones de venta, de esta manera asegurar su ingreso mensual para sostener a su familia."

\section{Segunda parte: reclutamiento, selección y rotación del personal de ventas}

En esta primera fase del método Delphi los expertos aportaron los 
siguientes comentarios: "Hay una por una necesidad de trabajo y no necesidad de desarrollar mayor y mejor necesariamente por vocación". Los gestión de liderazgo enfocada en resultados de esta parte de la desarrollo humano y del talento. Muchos investigación se encuentran reflejados en profesionales llegan a la función de venta las tablas 4 y 5 .

Tabla 4: Resultados de la primera ronda Delphi: reclutamiento, selección y rotación del personal de ventas

\begin{tabular}{|l|c|c|}
\hline Preguntas & Promedio & Mediana \\
\hline $\begin{array}{l}\text { Las empresas en el Perú usan una descripción de cargos para seleccionar al } \\
\text { personal de ventas. }\end{array}$ & 3,31 & 3 \\
\hline $\begin{array}{l}\text { La principal fuente de reclutamiento de personal de ventas son portales de } \\
\text { empleo }\end{array}$ & 3,44 & 3 \\
\hline $\begin{array}{l}\text { La principal fuente de reclutamiento de personal de ventas son referencias de } \\
\text { conocidos }\end{array}$ & 3,44 & 3 \\
\hline $\begin{array}{l}\text { La principal fuente de reclutamiento de personal de ventas son los } \\
\text { headhunters o cazatalentos }\end{array}$ & 2,75 & 3 \\
\hline $\begin{array}{l}\text { Las empresa en el Perú miden la rotación de vendedores } \\
\text { Las empresas en el Perú conocen las razones de la rotación de personal de } \\
\text { ventas }\end{array}$ & 3,38 & 3,5 \\
\hline \begin{tabular}{l} 
TOTAL \\
\hline
\end{tabular}
\end{tabular}

Fuentes: propias

Sobre esta primera fase Delphi no se pueden sacar conclusiones preliminares. Complementariamente, los expertos aportaron los siguientes comentarios "Las fuerzas de ventas suelen concentrarse en productos más fáciles debido a su rápida aceptación, aunque caen en el juego de precios, esto debido a la presión existente por el cumplimiento de metas. La diferencia en 
colocar productos más rentables suele pasar por la poca estrategia de comunicación o ausencia de nichos de mercado identificados para este tipo de productos. Sigue siendo importante el estudio de mercado y análisis de necesidades que realicen las áreas de Marketing y $\mathrm{BI}$.

Tabla 5: Resultados de la segunda ronda Delphi: reclutamiento, selección y rotación del personal de ventas

\begin{tabular}{|l|c|c|}
\hline Preguntas & Promedio & Mediana \\
\hline $\begin{array}{l}\text { Las empresas en el Perú usan una descripción de cargos para seleccionar al } \\
\text { personal de ventas. }\end{array}$ & 3,5 & 4 \\
\hline $\begin{array}{l}\text { La principal fuente de reclutamiento de personal de ventas son portales de } \\
\text { empleo }\end{array}$ & 3,33 & 4 \\
\hline $\begin{array}{l}\text { La principal fuente de reclutamiento de personal de ventas son referencias de } \\
\text { conocidos }\end{array}$ & 3,42 & 3 \\
\hline $\begin{array}{l}\text { La principal fuente de reclutamiento de personal de ventas son los } \\
\text { headhunters o cazatalentos }\end{array}$ & 2,75 & 2 \\
\hline $\begin{array}{l}\text { Las empresa en el Perú miden la rotación de vendedores } \\
\text { vas empresas en el Perú conocen las razones de la rotación de personal de }\end{array}$ & 2,75 & 3 \\
\hline \begin{tabular}{l} 
TOTAL \\
\hline
\end{tabular}
\end{tabular}

Fuentes: propias

Una vez realizada la segunda etapa del proceso en el apartado de reclutamiento, selección y rotación del personal de ventas, se puede extraer que la principal fuente de reclutamiento son las referencias de conocidos. Los headhunters 0 cazatalentos son escasamente usados. Tras dos rondas se puede concluir que en el Perú sí son usadas descripciones de cargo para reclutar y seleccionar vendedores. A su 
vez, que la rotación de fuerzas de ventas no es medida. Finalmente, y después de realizar el proceso de expertos respecto al tema de reclutamiento, selección y rotación del personal de ventas, no se concluir determinantemente que el reclutamiento y selección de los vendedores se desarrolla de manera sistemática. A su vez, la rotación se la considera como un hecho de la causa, y no se miden ni investigan sus causas. Fruto de los resultados de este apartado se puede confirmar la segunda hipótesis que el reclutamiento, selección y rotación de los vendedores no se desarrolla de manera sistemática ni planificada. En este ítem los expertos no aportaron sus comentarios.

\section{Tercera parte: desarrollo de competencia en ventas}

En esta primera fase sobre el desarrollo de competencia en ventas, los expertos aportaron los siguientes comentarios: "Las empresas transnacionales usan mucho el e-learning propio y brindan cursos de entrenamiento según sus programas de desarrollo de talento. Las empresas peruanas grandes y medianas brindan entrenamientos a su fuerza de ventas, pero no necesariamente con un plan de desarrollo bien definido. Las empresas pequeñas tienen limitaciones para brindar entrenamientos. Muchos vendedores optan por pagar ellos mismos sus cursos en diferentes instituciones y universidades". También "Muchas de las empresas mediana y pequeñas del Perú no tienen un sistema de evaluación de desempeño, solamente ven los resultados de venta. Los sistemas de salario consideran dos tipos: uno con un salario base más comisiones de venta y otro con un salario mensual más un bono por logro de objetivos, este último mantiene mucho en la zona de confort a los vendedores con poca ambición." Los resultados de esta parte de la investigación los encontramos reflejados en las tablas 6 y 7 . 
Tabla 6: Resultados de la primera ronda Delphi: desarrollo de competencia en ventas

\begin{tabular}{|l|c|c|}
\hline Preguntas & Promedio & Mediana \\
\hline Las empresas en el Perú tienen un proceso formal de capacitación de ventas & 3,06 & 3 \\
\hline Las empresas en el Perú usan capacitación corporativa para capacitar en ventas & 3,06 & 3 \\
\hline $\begin{array}{l}\text { Las empresas en el Perú usan programas abiertos en escuelas de negocios } \\
\text { para capacitar en ventas }\end{array}$ & 2,44 & 3 \\
\hline $\begin{array}{l}\text { Las empresas en el Perú usan programas cerrados en escuelas de negocios } \\
\text { para capacitar en ventas }\end{array}$ & 2,63 & 3 \\
\hline $\begin{array}{l}\text { Las empresas en el Perú capacitan en ventas a través de consultores } \\
\text { especialistas }\end{array}$ & 2,94 & 3 \\
\hline $\begin{array}{l}\text { Las empresas en el Perú miden la efectividad de sus programas de capacitación } \\
\text { de fuerzas de ventas }\end{array}$ & 2,63 & 2,5 \\
\hline $\begin{array}{l}\text { La capacitación de ventas está más orientada a vendedores que supervisores } \\
\text { TOTAL }\end{array}$ & $\mathbf{2 , 8 3}$ & $\mathbf{3}$ \\
\hline
\end{tabular}

Fuentes: propias

Sobre esta primera parte del miden la efectividad de la capacitación estudio sobre el desarrollo de que realizan. competencias en ventas solo se puede concluir preliminarmente que las empresas en el Perú mayormente no 
Tabla 7: Resultados de la segunda ronda Delphi: desarrollo de competencia en ventas

\begin{tabular}{|l|c|c|}
\hline Preguntas & Promedio & Mediana \\
\hline Las empresas en el Perú tienen un proceso formal de capacitación de ventas & 3 & 3 \\
\hline $\begin{array}{l}\text { Las empresas en el Perú usan capacitación corporativa para capacitar en } \\
\text { ventas }\end{array}$ & 2,41 & 2 \\
\hline $\begin{array}{l}\text { Las empresas en el Perú usan programas cerrados en escuelas de negocios } \\
\text { para capacitar en ventas }\end{array}$ & 2,31 & 2 \\
\hline $\begin{array}{l}\text { Las empresas en el Perú capacitan en ventas a través de consultores } \\
\text { especialistas }\end{array}$ & 2,83 & 2 \\
\hline $\begin{array}{l}\text { Las empresas en el Perú miden la efectividad de sus programas de } \\
\text { capacitación de fuerzas de ventas }\end{array}$ & 2,33 & $\mathbf{2}$ \\
\hline \begin{tabular}{l} 
La capacitación de ventas está más orientada a vendedores que supervisores \\
\hline TOTAL
\end{tabular} & $\mathbf{2 , 0 8}$ & 3,5 \\
\hline
\end{tabular}

Fuentes: propias

Después de realizar la segunda parte del proceso de expertos respecto al tema de desarrollo de competencias en ventas, no se puede extraer cuál es el tipo de proveedor con el cual las empresas capacitan a su canal de ventas, así como tampoco si las compañías tienen un proceso formal de capacitación. Sí se puede concluir que en general las empresas no miden la efectividad de sus programas de capacitación. Complementariamente, los expertos no aportaron comentarios.

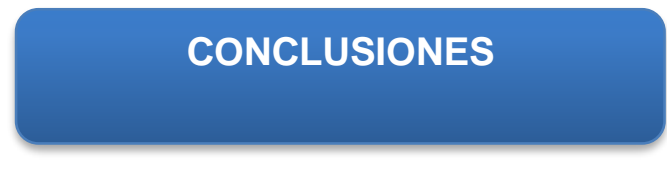

Se puede concluir que los supervisores de ventas en el Perú guían a sus dirigidos en oportunidades de ventas. No obstante, lo anterior, no destinan suficiente tiempo a reclutar y seleccionar vendedores. Por último, no facilitan el buen desarrollo de la cultura corporativa. Por lo que se puede afirmar la como válida la primera hipótesis en que los supervisores de ventas no gestionan bien 
sus roles relacionados a la gestión de los recursos humanos. La segunda hipótesis se confirma parcialmente, pues la rotación de fuerzas de ventas no es medida. Dado que las empresas en el Perú mayormente no miden la efectividad de la capacitación que realizan, y agregando los aportes de los expertos podemos dar como válida la tercera hipótesis en que las grandes empresas realizan una formación más planificada y sistemática que en las pequeñas y medianas empresas.

Esta investigación aporta un diagnóstico respecto a la gestión de los recursos humanos en las fuerzas de ventas considerando diversas variables tales como el reclutamiento y selección de comerciales, y el desarrollo de habilidades. En ese sentido, los resultados del trabajo demuestran que los supervisores de ventas no dedican el tiempo suficiente al reclutamiento y selección de vendedores ni al desarrollo de sus habilidades comerciales.

Respecto a las implicaciones gerenciales, se pueden indicar varios desafíos para la alta dirección, entre los que podemos mencionar: a) dar la debida importancia a los supervisores de ventas como un elemento clave para la mejora de la productividad comercial; b) tomar el desarrollo del liderazgo comercial y de habilidades de ventas como un proceso continuo y sistemático, y como un conductor relevante para generar ventajas competitivas; c) otro posible desafío para la dirección puede ser la creación, implementación y mejora de sus modelos de reclutamiento y selección del personal de ventas, donde cuenten con herramientas tales como un perfil profesional de sus supervisores y vendedores.

En relación a las limitaciones de esta investigación se puede mencionar que el hecho de tener en cuenta la perspectiva de un panel de expertos limitado en número y de perfiles diversos puede producir sesgos. Por lo que estos resultados podrían ser contrastados de manera complementaria con las percepciones de clientes finales $y / 0$ proveedores.

Dentro de las futuras líneas de investigación se extrae el estudio de mejores prácticas y modelos de gestión de las fuerzas de ventas que logren la creación de valor para clientes, vendedores y la propia compañía. A su vez, sería interesante poder entender y 
explicar las razones de las bajas valoraciones relativas que este trabajo ha arrojado, como también poder comparar estos resultados dentro de distintos sectores industriales en el Perú, y en países de Hispanoamérica. Por último, conocer las mejores prácticas tales como la aplicación de sistemas tecnológicos por sectores industriales del Perú y en otros países.

\section{BIBLIOGRAFÍA}

Albers, S., Mantrala, M. K., \& Sridhar, S. (2010). Personal selling elasticities: A meta-analysis. Journal of Marketing Research, 47(5), 840-853.

Attia, A. M., Honeycutt, E. D. \& Fakhr R. (2012). Sales Training Evaluation: An Integrated Framework and Research Agenda. Journal of Selling \& Major Account Management, 13(1), 33-44.

Baldauf, A. \& Lee, N. (2011). International Selling and Sales Management: Sales Force Research beyond Geographic Boundaries. Journal of Personal Selling \& Sales Management, 31 (3), 211-217.

Barat, S., \& Spillan, J. E. (2009). A CROSS COUNTRY COMPARATIVE ANALYSIS OF STUDENTS'PERCEPTIONS OF THE SALES PROFESSION: A LOOK AT PERU, GUATEMALA, AND US. Marketing Management Journal, 19(2).

Basu, S. \& Schroeder R. G. (1977). Incorporating Judgments in Sales Forecasts: Application of the Delphi Method at American Hoist \& Derrick. Interfaces. 3.18-27

Bolander, W., Dugan, R., \& Jones, E. (2017). Time, change, and longitudinally emergent conditions: understanding and applying longitudinal growth modeling in sales research. Journal of Personal Selling \& Sales Management, 37(2), 153159.

Bonnemaizon, A., Cova, B., \& Louyot, M. C. (2007). Relationship marketing in 2015: a Delphi approach. European Management Journal, 25(1), 50-59. 
Cabero Almenara, J., \& Infante Moro, A. (2014). Empleo del método Delphi y su empleo en la investigación en comunicación y educación. Edutec, 48, 1-16.

Calabuig, F. \& Crespo, J. (2009). Uso del método Delphi para la elaboración de una medida de la calidad percibida de los espectadores de eventos deportivos. RETOS. Nuevas tendencias en Educación Física, Deporte y Recreación, 15, 21-25.

Campbell, J. B., \& Fransi, E. C. (2016). Principales problemas en la gestión y dirección de ventas en Chile: estudio a través del método Delphi. Revista Academia \& Negocios, 2(2), 1-16.

Campbell, J. B., \& Fransi, E. C. (2016). ESTUDIO SOBRE EL ESTADO DEL RECLUTAMIENTO $Y$ SELECCIÓN FUERZAS DE VENTAS A TRAVÉS DE UN PANEL DE EXPERTOS EN CHILE. Neumann Business Review, 2(2), 48-71.
Campos, V., Melián A., Sanchis J. R. (2014). El método Delphi como técnica de diagnóstico estratégico. Estudio empírico aplicado a las empresas de inserción en España. Revista Europea de Dirección y Economía de la Empresa. 23(2). 72-81.

Cerasoli, C. P., Nicklin, J. M., \& Ford, M. T. (2014). Intrinsic Motivation and Extrinsic Incentives Jointly Predict Performance: A 40-Year MetaAnalysis. Psychological Bulletin. 140 (4). 980-1008.

Chang, P. C. \& Wang Y. W. (2006). Fuzzy Delphi and back-propagation model for sales forecasting in PCB industry. Expert Systems with Applications. 30(4). 715-726.

Chung, D. J., Steenburgh, T., \& Sudhir, K. (2013). Do bonuses enhance sales productivity? A dynamic structural analysis of bonus-based compensation plans. Marketing Science, 33(2), 165-187.

Cron, W. Marshall, G.W. Singh, J. Spiro, R., \& Sujan, H. (2005). Salesperson selection, training, 
and Development: trends, implications, and research Opportunities. Journal of Personal Selling \& Sales Management, 25 (2), 123-136.

Darmon, R. (2008). The Concept of Salesperson Replacement Value: A Sales Force Turnover Management Tool. Journal of Personal Selling \& Sales Management, 28 (3), 211-232.

Dawson, J. A. (1994). Internationalization of retailing operations. Journal of marketing management, 10(4), 267-282.

Deconinck, J., Johnson J. \& Busbin J. (2012). The Effect of Organizational Justice on Salespersons' Perceived Ethical Climate, Organizational Commitment and Turnover Intentions. Journal on Business Review, 2 (2), 57-64.

Farrell, S. Hakstian, A. (2001). Personnel Selection Procedures and Training Interventions. Psychology \& Marketing, 18(3), 281-316.
Flores, M. A. (2012). Gestión de ventas en un establecimiento farmacéutico de la cadena de Boticas Arcángel de la ciudad de Chiclayo durante abril a julio del 2012.

Gil-Gómez de Liaño, B. \& Pascual-Ezama, D. (2012). La metodología Delphi como técnica de estudio de la validez de contenido. Anales de Psicología, 28 (3), 1011-1020.

Gupta, U. G. \& Clarke R. E. (1996). Theory and applications of the Delphi technique: A bibliography (19751994). Technological Forecasting and Social Change, 53, 185-211.

Gutierrez, A. (2015). Análisis del retorno de la inversión en la gestión comercial de los supermercados en el Perú.

Hasson, F., Keeney, S., \& McKenna, H. (2000). Research guidelines for the Delphi survey technique. Journal of advanced nursing, 32(4), 1008-1015.

Hayes, T. (2007). Delphi study of the future of marketing of higher education. Journal of Business Research, 60(9), 927-931. 
Hill, J. S., Still, R. R., \& Boya, Ü. O. (1991). Managing the multinational sales force. International Marketing Review, 8(1).

Hill, J. S., \& Still, R. R. (1990). Organizing the overseas sales force: how multinationals do it. Journal of Personal Selling \& Sales Management, 10(2), 57-66.

Hill, J. S., \& Birdseye, M. (1989). Salesperson selection in multinational corporations: an empirical study. Journal of Personal Selling \& Sales Management, 9(2), 39-47.

Hill, J. S., \& Allaway, A. W. (1993). How US-based companies manage sales in foreign countries. Industrial Marketing Management, 22(1), 7-16.

Jaramillo, F., Mulki J. P. \& Boles J. S. (2013). Bringing meaning to the sales job: The effect of ethical climate and customer demandingness. Journal of Business Research, 66 (11), 2301-2307.
Jiang, K., Lepak D. P., Han K., Hong Y., Kim A. \& Winkler A. L. (2012). Clarifying the construct of human resource systems: Relating human resource management to employee performance. Human Resource Management Review, 22 (2), 73-85.

Karakaya, F., Quigley, C., \& Bingham, F. (2011). A cross-national investigation of student intentions to pursue a sales career. Journal of Marketing Education, 33(1), 1827.

Kauko, K. \& Palmroos P. (2014). The Delphi method in forecasting financial markets- an experimental study. International Journal of Forecasting, 30 (2), 313-327.

Khosla, R., Goonesekera, T., \& Chu, M. T. (2009). Separating the wheat from the chaff: An intelligent sales recruitment and benchmarking system. Expert Systems with Applications, 36(2), 3017-3027.

Kim, Y., \& Talbott, J. S. (2018). Marketing social selling jobs: a re-labelling 
strategy. Marketing Intelligence \& Planning, 36(1), 2-16.

Loo, R. (2002). The Delphi method: a powerful tool for strategic management. Policing an International Journal of Police Strategies and Management, 25(4), 762-769.

Magnusson, P., Peterson R. \& Westjohn S. A. (2014). The influence of national cultural values on the use of rewards alignment to improve sales collaboration. International Marketing Review, 31, 30-50.

Mantrala, M. K., Albers, S., Caldieraro, F., Jensen, O., Joseph, K., Krafft, M., \& Lodish, L. (2010). Sales force modeling: State of the field and research agenda. Marketing Letters, 21(3), 255-272.

Misra, S., \& Nair, H. S. (2011). A structural model of sales-force compensation dynamics: Estimation and field implementation. Quantitative Marketing and Economics, 9(3), 211-257.
Mitra, S. (2013). Scenario Generation for Operational Risk. Intelligent Systems in Accounting, Finance and Management. 20 (3), 163187.

Nielson, B., Eichmeier, S., \& CooperLarsen, D. (2017). Organizational Compensation Limitations Inhibiting Recruitment of Top-Tier Qualified Sales Graduates. International Journal of Sales, Retailing \& Marketing, 6(1).

Nijs, S., Gallardo E., Dries N. \& Sels L. (2013). A multidisciplinary review into the definition, operationalization, and measurement of talent. Journal of World Business, 49 (2), 180-191.

Powers, T. L., DeCarlo T. E. \& Gupte G. (2010). An update on the status of sales management training. Journal of Personal Selling \& Sales Management, 30 (4), 319326.

Rojas, P., \& Tito, A. (2015). Implementación de una aplicación web de gestión de ventas e inventarios en la Empresa Inversiones Huaytatex SA para 
controlar el proceso de toma de decisiones.

Rowe, G. \& Wright G. (1999). The Delphi technique as a forecasting tool: issues and analysis. International Journal of Forecasting. 15 (4). 353-375.

Saeed, A., Zehou S., Hussain N. \& Anwar ul Haq M. (2014). Customer Satisfaction and Shareholder's Value: The Role of Employee Satisfaction. European Journal of Business and Management, 6 (13), 198-203.

Sager, J. K., Dubinsky A. J., Wilson P. H. \& Shao C. (2014). Factors Influencing the Impact of Sales Training: Test of a Model. International Journal of Marketing Studies, 6 (1), 1-20.

Småros, J. \& Hellström M. (2004). Using the assortment forecasting method to enable sales force involvement in forecasting. International Journal of Physical Distribution \& Logistics Management. 34 (2), 140-157.
Taleghani, M., Biabani, S., Gilaninia, S., Rahbarinia, S., \& Mousavian, S. (2011). The Relationship between Customer Satisfaction and Relationship Marketing Benefits. Arabian Journal of Business and Management Review, 1(3), 7886.

Vélez, I. (2002). Decisiones de inversión enfocado a la valoración de empresas. Bogotá, Colombia: CEJA.

Wang, Y. \& Hsieh H. (2012). Toward a Better Understanding of the Link Between Ethical Climate and Job Satisfaction: A Multilevel Analysis. Journal of Business Ethics, 105 (4), 535-545.

White, D. W., Absher, R. K., \& Huggins, K. A. (2011). The effects of hardiness and cultural distance on sociocultural adaptation in an expatriate sales manager population. Journal of Personal Selling \& Sales Management, 31(3), 325-337.

Wood, L. \& Mauskopf J. A. (1991). Forecasting market potential and 
market penetration of residential water heater load control programs. Journal of Forecasting 10 (4), 399-413.

Zoltners, A., Sinha P. \& Lorimer S. E. (2012). Breaking the sales force incentive addiction: a balanced approach to sales force effectiveness. Journal of Personal Selling \& Sales Management, 32 (2), 171-186.

Zoltners, A., Sinha P. \& Lorimer S. E. (2008). Sales Force Effectiveness: A Framework for Researchers and Practitioners. Journal of Personal Selling \& Sales Management, 28 (2), 115131. 